Military Technical College
Kobry Elkobbah,
Cairo, Egypt

\title{
ANALYSIS OF THE QUEUEING BEHAVIOR OF AUTOMATIC ESM SYSTEMS
}

\author{
M. H. El-Ayadi*, K. El-Barbary"* and H. E. Abou-Bakr**
}

\begin{abstract}
Radar electronic support measures (ESM) system are responsible of threat detection and area surveillance to determine the identity and bearing of the surrounding active emitters. The high arrival rate of radar pulses in dense emitter environments demands fast automatic processing of arriving pulses so that the ESM system can fulfill its functions properly in real time. Yet, the performance analysis of automatic ESM system is usually encountered with the difficulty that both pulse arrivals and widths can be specified only probabilistically $[1,2,3]$. The success of Queueing theory $[4,5]$ in many applications such as computer communication networks and flowcontrol has encouraged designers to utilize queueing theory in qualifying and judging the performance of automatic ESM systems in dense emitter environments. The queueing behavior of these systems is analytically evaluated in this paper under different service disciplines and the results are validated by elaborating computer simulations. The analysis involves statistical modeling of arrival and departure processes as well as distribution of service times. It permits estimating the blocking probability due to high arrival rates of intercepted radar pulses or due to linited speed of the deinterleaver processor. The analysis also indicates some system configuration trade-offs.
\end{abstract}

\section{KEY WORDS}

Radar pulses, deinterleaver algorithms, sorting and radar cells.

\section{NOMENCLATURE}

PDV : Pulse descriptor vector

EDV : Emitter descriptor vector

Professor of signals \& systems - Faculty of Computer and Information Sciences, Ain Shams University, Cairo, Egypt.

"Egyptian Armed Forces. 


\section{INTRODUCTION}

Early radar electronic support measures (ESM) systems relied on human operator interpretation of ESM receiver output to provide classification and identification of intercepted emitters. The steadily increasing density of radar pulse environments leads to the requirement of some form of automatic ESM processing to cope with

system can be divided into two main subsystems:(1) the receiver-encoder subsystem, that measures the parameters of each received radar pulse and encodes them by a digital word called pulse descriptor vector (PDV), and (2) the deinterleaver processor, that rapidly sorts the PDVs into chains each comprising a group of PDVs supposed to be emitted from the same radar. This paper analyzes the queueing behavior of automatic ESM systems in dense emitter environments.

Queueing theory is concerned with the abstract mathematical modeling of systems subject to demands whose occurrences and lengths can, in general, be specified only probabilistically. Although, these systems are usually very complex, it is often possible to abstract from the system description a mathematical model whose analysis yields useful information about the quality of the service and the efficient utilization of the system. In this paper, reception of radar pulses, and extraction of their parameters as well as sorting of PDVs into separate chains is modeled as a finite-state machine operating as a queue with a single server. It is known that the application of the queueing theory is possible in principle if both the arrival process at the queueing model input and the service time distribution inside it are statistically characterized. All queueing models discussed in this paper are conventionally labeled as $[4,5]$ :

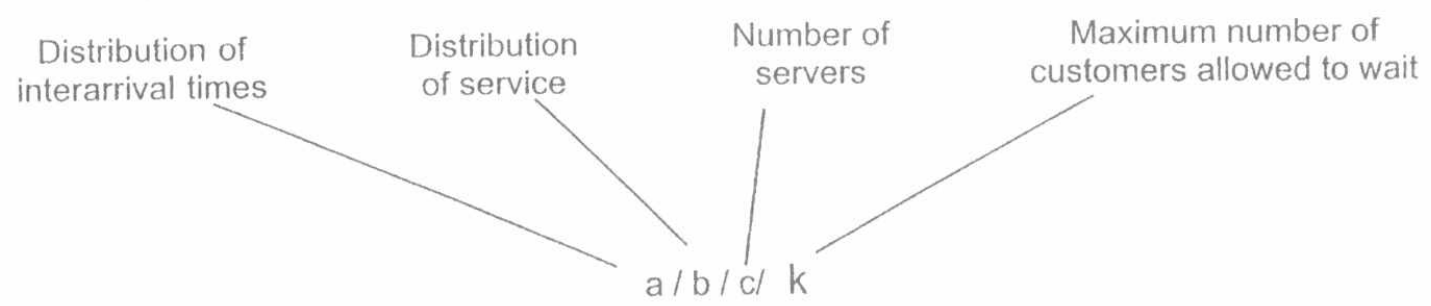

\section{THE QUEUEING MODEL REPRESENTATION OF ESM RECEIVER-ENCODER SUBSYSTEM}

\section{Distribution of interarrival times:}

In dense emitter environment, the interarrival time between successive pulses at the ESM receiver input is a random variable distributed according to a negative exponential distribution with parameter $\lambda$ equal to the sum of the PRFs of all active radars in the instantaneous view of ESM receiver[6]. Since for negative exponential distribution $1 / \lambda$ is the mean value (of interarrival time), then $\lambda$ is also the average arrival rate of radar pulses. As the number and PRF diversity of active pulse emitters increase the arrival process at the input of ESM receiver tends to be a stationary Poisson process. That is given a time interval $\tau$, the probability that exactly $n$ pulses arrive at the ESM receiver input during $\tau$ is

$$
P_{n}(\tau)=e^{-\lambda \tau} \frac{(\lambda \tau)^{n}}{n !}
$$


The average arrival rate was measured through computer simulations of different number of active emitters with different PRFs. Both the calculated arrival rate $\lambda$ and the measured one $\lambda_{\text {measured }}$ are provided in Table 1.Clearly, the arrival rate of radar pulses at the ESM receiver input is directly proportional to the number of active radars illuminating the ESM receiver, and equals to the sum of PRFs of these radars. The values of the interarrival time between successive received radar pulses were observed over a long interval. The moments of the interarrival time up to the fourth order were computed and denoted as $M_{i_{m}} ; 1 \leq \mathrm{i} \leq 4$. The first four moments $M_{i_{t}} ; 1 \leq \mathrm{i}$ $\leq 4$ of the negative exponential distribution with parameter $\lambda$ taken to be the sum of PRFs of simulated active radars were calculated. We defined a normalized squared distance $D^{2}$ between the theoretical and the measured densities of the interarrival time as

$$
D^{2}=\left\|\begin{array}{l}
M_{1 t}-M_{1 m} \\
M_{2 t}-M_{2 m} \\
M_{3 t}-M_{3 m} \\
M_{4 t}-M_{4 m}
\end{array}\right\|^{2} / \|\left(\begin{array}{l}
M_{1 t} \\
M_{2 t} \\
M_{3 t} \\
M_{4 t}
\end{array} \|^{2}\right.
$$

where, $\|x\|$ denotes the norm of the vector $\mathbf{x}$. The results are presented in Table 2 for different simulated arrival rates of radar pulses emitted from the active radars in the instantaneous view of ESM receiver. From Table 2, it is clear how close the measured distribution is to the theoretical one. For the data in Table 2, $0.26 \times 10^{-6} \leq$ $\mathrm{D}^{2} \leq 5.54 \times 10^{-5}$.

The third property, we verify, is that the number of received pulses at the ESM receiver input during any observation time is a randomly distributed according to Poisson distribution with parameter $\lambda$. Thus, we simulate an instantaneous field of view with $\lambda=30000$ pulse $/ \mathrm{sec}$. and observation time $=0.21 \mathrm{sec}$. Then, we divide this observation time into a number of sub-intervals $L$. We count the number of pulses $n$ occurring in each sub-interval. We evaluate the probability of every counted $n$. Finally, we calculate the corresponding probabilities from Poisson distribution and compare these probabilities with the measured ones. We repeat the above procedure for different lengths of the sub intervals as shown in Table 3.

\section{Distribution of service times:}

The distribution of service time inside the ESM receiver depends on what pulse parameters are measured and on the service discipline of the receiver-encoder subsystem[1,2]. Generally, there are two service disciplines of an ESM receiverencoder [1,2], namely the paralayzable counter with constant dead-time and the nonparalayzable counter. Both service disciplines do not allow waiting. Under the paralayzable service discipline, an ESM receiver-encoder [1,2] can only process pulse which arrives after fixed time $\tau$ from the previous received pulse. The corresponding queueing model is the $\mathrm{M} / \mathrm{D} / 1 / 0$ model $^{(1)}[4,5]$. Typical values of fixed service time are the mean and the maximum widths of arriving pulses. Under the non-paralayzable counter service discipline an ESM receiver-encoder is ready to process a new coming pulse as soon as the previous pulse is expired. Thus the service time for each received pulse is equal to its width.

\footnotetext{
(1) The $M / D / 1 / k$ model assumes that all service times have actually equal fixed values (deterministic values) and that we have a Poisson input process with fixed mean arrival rate, only one server and maximum number of customers allowed to wait is $\mathrm{k}$.
} 
For overlapping pulses the service time is the maximum of the resultant width of overlapped pulses and certain maximum permissible value [7]. This avoids overloading by a CW signal.

The distribution of the service time under paralayzable counter service discipline is given by $\delta(t-\tau)$ and under the non-paralayzable counter discipline is given by the actual distribution of the widths of arriving radar pulses. A good statistical model of the service time is the Erlang distribution with parameters $\theta$ and $\mu[4,5]$,

$$
\begin{aligned}
& f(t)=(\theta \mu)^{\theta} t^{\theta-1} \frac{e^{-\mu \theta}}{\theta !}, \\
& E\{t\}=\frac{1}{\mu}, \\
& \sigma=\sqrt{E\left\{t^{2}\right\}-E^{2}\{t\}}=\frac{1}{\mu \sqrt{\theta}}, \\
& \frac{\sigma}{E\{t\}}=\frac{1}{\sqrt{\theta}} .
\end{aligned}
$$

Notice that for $\theta=1$, the Erlang distribution reduces to the exponential distribution. This would be the distribution of the service time under the non-paralayzable service discipline when the main contributors to the arrival process at the input of ESM system are short pulse radars, (like range finders or tracking radars). For $\theta>1$, the mode of Erlang distribution shifts rightward from zero and the coefficient of variation $\sigma / E\{t\}$ decreases. This might approximate the distribution of the service time under the non-paralayzable counter service discipline in dense environment of surveillance radars with low diversity of pulse widths. Ideally, the distribution of widths of arriving pulses should be a multimodal distribution with the number of modes corresponding to the number of active pulse radar emitters. But assuming low diversity of widths and considering multi-path and narrowband filtering effects the actual distribution might be a unimodal distribution. Finally, as $\theta \rightarrow \infty$, the Erlang distribution tends to the deterministic one $\delta(t-1 / \mu)$. Thus the queueing model of an ESM receiver Linder non-paralayzable counter service discipline is $M / G / 1 / 0^{(1)},[4,5]$.

\section{EVALUATION OF THE FREE STATE PROBABILITY $P_{f}(t)$}

Under either service disciplines the ESM receiver-encoder is usually in one of the following two states:

1-Free state, where the ESM receiver-encoder is free and ready to receive any new coming pulse. The probability that the ESM receiver is free is denoted by $P_{f}(t)$.

2-Busy state, where the system is busy processing a pulse and cannot receive and process another pulse. So, in this state the new coming pulse will be lost. The probability that the receiver is busy is denoted by $P_{b}(t)$.

Since the system must be in one of the above states, it is evident that

$$
P_{f}(t)+P_{b}(t)=1
$$

The ESM receiver-encoder will be in the free state at time instant $t+\Delta t$ if either of the events $e_{1}$ or $e_{2}$ happens:

\footnotetext{
(1) The M/ G/ 1/ 0 assumed that the queueing system has a single server, maximum number of customers allowed to wait is zero and a Poisson input process (exponential interarrival times). The customers are assumed to have independent service times but with the same (general) probability distribution..
} 
$\mathrm{e}_{1}$ : The ESM receiver-encoder is free at the instant $\mathrm{t}$ and no pulse arrives during the interval $\Delta \mathrm{t}$.

$\mathrm{e}_{2}$ :The ESM receiver-encoder is busy at time instant $t$, processing of current radar pulse terminates during the subsequent interval $\Delta t$ and no new pulse arrives.

From (1) we have

$$
\begin{aligned}
& P_{n=0}(\Delta t)=e^{-\lambda \Delta t} \\
& P\left(e_{1}\right)=P_{f}(t) e^{-\lambda \Delta t} \approx P_{f}(t)(1-\lambda \Delta t)
\end{aligned}
$$

In [7], it is shown that the probability $\mathrm{P}\left(\mathrm{e}_{2}\right)$ can be expressed in the form of $\mu \Delta t$, no matter the service discipline adopted and the distribution of the service time. Table 4 provides expressions for $\mu$ in four different cases. From the above presentation, we can express $P_{f}(t+\Delta t)$ as

$$
P_{f}(t+\Delta t)=P_{f}(t)(1-\lambda \Delta t)+\mu \Delta t
$$

which reduces to a first order differential equation

$$
P_{f}^{\prime}(t)+\lambda P_{f}(t)=\mu
$$

The steady state solution of the above equation is

$$
P_{f}(t)=\mu / \lambda
$$

Substituting from Table 1 into (10) we get

$$
\begin{array}{ll}
\text { Case 1: } & P_{f}(t)=e^{-\lambda \tau} \\
\text { Case 2: } & P_{f}(t)=\frac{1}{1+\lambda \bar{w}} \\
\text { Case 3: } & P_{f}(t)=\left(1+\frac{\lambda \bar{w}}{\theta}\right)^{-\theta} \\
\text { Case 4: } & P_{f}(t)=\frac{e^{-\lambda w_{\min }}-e^{-\lambda w_{\max }}}{\lambda\left(w_{\max }-w_{\min }\right)}
\end{array}
$$

Fig. 1 compares the ratios of successfully processed pulses under paralayzable service discipline (case e) and the same ratio under non-paralayzable service discipline cases $(a, b, c, d)$. Note that $M / E_{\infty} / 1 / 0 \equiv M / D / 1 / 0$ with $\tau=1 / \mu$. It is assumed that successfully processed pulses are only these completely received. Fig. 1 indicates that for a given arrival rate, as the parameter $\theta$ of the Erlang distribution increases, the ratio of successfully processed pulses under nonparalayzable service discipline decreases. But, for all finite $\theta$ it remains higher than the same ratio under paralayzable service discipline. Yet in practice, there can exist dense emitter environments providing pulse arrival rates much higher than those presented in Fig. 1. Clearly, at such very high arrival rates the ratio of successfully processed pulses will be extremely low. Possible cures are either to equip the ESM system with several receiving-encoding channels, (multiple servers), or to limit the measurements of mono pulse parameters to those that can be obtained from the pulse leading edge and imposing the paralayzable service discipline. In this case the fixed service time is of order $0.1 \mu \mathrm{sec}$. or even $0.05 \mu \mathrm{sec}$. From (13-a), the ESM receiver -encoder will be able to process up to $10^{6}$ pulse/sec. with success ratio $90 \%$. 


\section{THE ESM RECEIVER-ENCODER DEPARTURE PROCESS}

Under the paralayzable counter service discipline, the departure process is a renewal process since the pulses of the input flow which are blocked are not randomly selected. The departure process has therefore a residual effect and is not a Poisson process. The distribution of the interdeparture times between successive processed pulses $f_{T d}(l)$ is deduced from the interarrival times of the input flow of pulses as follows

$$
\begin{aligned}
& f_{T d}(l) \Delta l=\frac{\{\text { the proportion of the distribution of the interarrival times in the range }(l, l+\Delta l)\}}{\{\text { the proportion of the distribution of the interarrival times in excess of } \tau\}} \\
& \qquad=\frac{\lambda e^{-\lambda \Delta l} \Delta l}{e^{-\lambda \tau}}, l>\tau \\
& \qquad f_{T d}(l)=\lambda \exp (-\lambda(l-\tau)) ; \quad l \geq \tau
\end{aligned}
$$

where, $T_{d}$ is the interdeparture time between output PDVs from the receiver-encoder subsystem and $\tau$ is the fixed service time of the receiver. We evaluated the sqiared distance between the theoretical and the measured densities of the interdeparture times, according to (2). The results are given in Table 5 for different simulated arrival rates. For data in Table $5,0.29 \times 10^{-4} \leq \mathrm{D}^{2} \leq 6.57 \times 10^{-4}$

Now we are going to show that under non-paralayzable counter service discipline if the arrival process is a Poisson process, then so is the departure process. Yet it is necessary that the PW of intercepted pulses be exponentially distributed or follow an Erlang distribution with moderate $\theta$.

Suppose that $n$ pulses arrive at the input of the ESM receiver during $L$ seconds and only $d$ of them are successfully processed by the ESM receiver-encoder subsystem and passed to the deinterleaver processor in the form of $d$ PDVs. Thus, $n-d$ pulses aren't processed or are missed. As indicated in the previous section each arriving pulse will with probability $P_{f}$ find the ESM receiver in free state and with probability $P_{b}$ in busy state. Hence, the number $d$ of pulses successfully processed out of $n$ pulses arriving during the interval $\mathrm{L}$ follows the binomial distribution,

$$
P_{d / n}(L)=\left(\begin{array}{l}
n \\
d
\end{array}\right) P_{f}^{d} P_{b}^{n-d}, 0 \leq d \leq \mathrm{n}
$$

where $P_{d / n}(L)$ is the probability that d PDVs will emerge from the ESM receiverencoder subsystem given that $n$ pulses are received at its input during time $L$.

Let us recall that in dense environments the number of pulses arriving at the ESM receiver input is a random variable distributed according to Poisson distribution with parameter $\lambda$. So, an expression for $P_{d}(L)$ which is the probability that $d$ PDVs will emerge from the receiver-encoder in $L$ seconds regardless of the number of arriving pulses, we use the law of total probability ; i .e.

$$
\begin{aligned}
P_{d}(L) & =\sum_{n=d}^{\infty} P_{d / n}(L) P_{n}(L), \\
& =\sum_{n=d}^{\infty}\left(\begin{array}{l}
n \\
d
\end{array}\right) P_{f}^{k} P_{b}^{n-d} P_{n}(L)
\end{aligned}
$$

Substitution of (1) into (17) gives 


$$
P_{d}(L)=\frac{\left(\lambda_{0} L\right)^{d}}{d !} e^{-\lambda_{0} t}, \mathrm{~d} \geq 0
$$

where,

$$
\lambda_{0}=\lambda P_{\mathrm{f}}
$$

From (18) it is clear that under the assumptions indicated above the departure process will be a Poisson process with departure rate $\lambda_{0}=\lambda P_{f}$. Consequently, the distribution of the times between successive output PDVs from the receiver-encoder subsystem is a negative exponential distribution with parameter $\lambda_{0}=\lambda P_{f}$. Computer simulations verified these conclusions and the results are presented in Table 6, where $0.017 \times 10^{-4} \leq \mathrm{D}^{2} \leq 11 \times 10^{-4} 0.017$.

If the assumption made on the distribution of widths of arriving pulses is absent, i.e. if for example $0<w_{\min } \leq P W \leq w_{\max }$, then the values of $d$ in (16) - (18) should be restricted to the following ranges:

- in (16), $\left[\mathrm{L} / \mathrm{w}_{\max }\right] \leq \mathrm{d} \leq \min \left\{\left[\mathrm{L} / \mathrm{w}_{\min }\right], \mathrm{n}\right\}$

- in (17) $\left[\mathrm{L} / \mathrm{W}_{\max }\right] \leq \mathrm{d} \leq\left[\mathrm{L} / \mathrm{W}_{\min }\right]$,

where, $[x]$ denotes the integral part of $x$. Obviously in this case the departure process is no longer a Poisson process since (18) is not valid for all $d, L \geq 0$.

\section{THE DEINTERLEAVER REPRESENTATION AS A QUEUEING MODEL}

The deinterleaver is modeled as a single server receiving a random stream of PDVs from the receiver-encoder subsystem. The service-time in the deinterleaver is the time needed to decide for a new arriving PDV which of the already formed chains, it should be attached to. Hence, its probability density function $b(t)$ is given by

$$
b(t)=P(1) \delta\left(t-t_{\text {comp }}\right)+P(2) \delta\left(t-2 . t_{\text {comp }}\right)+\cdots \cdots+\left[P(N)+P_{\text {new }}\right] \delta\left(t-N \cdot t_{\text {comp }}\right)
$$

where, $t_{\text {comp }}$ is the time needed of comparing a new PDV with one radar chain, $N$ is the number of chains formed inside the deinterleaver, $P(i), 1 \leq \mathrm{i} \leq \mathrm{N}$, is the probability that the incoming PDV matches the $\mathrm{i}^{\text {th }}$ radar chain, and $P_{\text {new }}$ is the probability thit the incoming PDV doesn't match any radar chain. If $P_{\text {new }}=0$, i. e. if $N$ is the total number of active emitters within the instantaneous view of the ESM receiver, then it can be shown that

$$
P(i)=\frac{P R F_{i}}{\sum_{i=1}^{N} P R F_{i}}, 1 \leq \mathrm{i} \leq \mathrm{N},
$$

The distributions of the service time inside the deinterleaver and of the interarrival times between received PDVs at the deinterleaver input are necessary to describe the queueing behavior of the deinterleaver. With the ESM receiver-encoder under the paralayzable counter service discipline, the deinterleaver queueing model will be the $\mathrm{f}_{\mathrm{Td}} / \mathrm{b} / 1 / 0 \quad[4,5]$, where $\mathrm{f}_{T d}$ is given by (15) and the density $b(t)$ is given by (20). But with the ESM receiver-encoder under the non-paralayzable counter service discipline and assuming exponentially distributed widths of intercepted pulses, the deinterleaver queueing model will be $\mathrm{M} / \mathrm{b} / 1 / 0 \quad[4,5]$. The analysis of the queueing behavior of the deinterleaver in latter case is simpler than in the former one. It allows to estimate the blocking (congestion) probability of the deinterleaver, i.e. the probability that a PDV arrives at the deinterleaver when it is busy processing another one.

It is important to note that the performance of the deinterleaver in terms of the blocking probability is much improved when it is preceded by a pre-buffer of size K. The function of this pre-buffer is to store up to $K$ of the arriving PDVs when the 
deinterleaver processor is busy. However, if a new PDV arrives while the deinterleaver pre-buffer is full, this PDV will be lost. The queueing model in this case is $\mathrm{M} / \mathrm{b} / 1 / \mathrm{K}$.

Suppose that the deinterleaver is preceded by a pre-buffer of size K. The instant at which the deinterleaver completes servicing the $n^{\text {th }}$ PDV is the instant when the deinterleaver is ready to service the $(n+1)^{\text {rst }}$ PDV. Let us designate such instant by $t_{n}$, $n=1,2,3 \quad$ and the corresponding state of the pre-buffer by the positive integer $k$, where $k$ is the number of PDVs left in the pre-buffer after the last processed PDV. Now we denote the probability that the pre-buffer is at instant $t_{n}$ in state $k$ by $\pi_{k}\left(t_{n}\right), 0$ $\leq \mathrm{k} \leq \mathrm{K}$. Assume that the input flow of PDVs is a Poisson process with average rate $\lambda_{0}$. The probability that $j$ PDVs arrive at the pre-buffer input during the service time of PDV number $n$ is

$$
r_{j}=P\left(\zeta_{n}=j\right)=\int_{0}^{\infty} P\left(\zeta_{n}=j, t\right) d t=\int_{0}^{\infty} \frac{e^{-\lambda_{0} t}\left(\lambda_{0} t\right)^{j}}{j !} b(t) d t
$$

where, $\zeta_{n}$ is a random variable denoting the number of PDVs arriving at the prebuffer input while servicing the PDV number $n$ and $b(t)$ is given by (20). The probabilities $r_{j}$ are transition probabilities associated with occurrence of different prebuffer states during servicing successive PDVs. It can easily be deduced that

$$
\pi_{j}\left(t_{n}\right)=\sum_{i=0}^{j} \pi_{j-i+1}\left(t_{n-1}\right) r_{i}, j=0,1,2
$$

As $\mathrm{n} \rightarrow \infty$, the system composed of the pre-buffer and the deinterleaver reaches a steady state and the pre-buffer state probabilities $\pi_{k} 0 \leq k \leq K$ become time independent. In particular, the steady state blocking probability will be given by the limit of $\pi_{k}\left(t_{n}\right)$ as $n \rightarrow \infty$. Moreover, the system (23) will take the form

$$
\sum_{i=0}^{j} \pi_{j-i+1}\left(r_{i}-\delta_{i, 1}\right)=0, \mathrm{j}=0,1,2
$$

where, $\delta_{i, 1}$ equals 1 if $i=1$ and equals zero for $i \neq 1$. The above system can be efficiently solved for $\pi_{1}, \pi_{2}, \pi_{3} \quad \pi_{K}$ in terms of $\pi_{0}$, the forward substitution algorithm [8]. By imposing the constraint $\sum_{i=0}^{K} \pi_{i}=1$ we can determine the value of $\pi_{0}$ and hence the values of the other state probabilities $\pi_{\mathrm{i}}, 1 \leq \mathrm{i} \leq \mathrm{K}$. Evidently, the number of bits used to generate the PDV will determine the value of the comparison time inside the deinterleaver. Thus, the longer the length of the PDV, the higher the blocking probability of the deinterleaver processor. So, if we desire to decrease the comparison time, the number of bits used to represent the PDV should be decreased, but this degrades the resolution and accuracy of measuring the parameters of the intercepted pulses. To maintain good measurement performance of each parameter without increasing the blocking probability of the deinterleaver we have two alternate solutions. The first one is to increase the pre-buffer size and the second is to choose a faster processor to decrease the comparison time. Figs. 2 and 3 show quantitatively the effect of the speed of the deinterleaver processor and the size of the pre-buffer on the blocking probability of the deinterleaver at different arrival rates of PDVs. Fig. 2 shows that for a fixed pre- buffer size and fixed average arrival rate of PDVs, the higher the speed of the deinterleaver processor, the lower the blocking probability. Also, the blocking probability is much reduced by increasing the size of the pre-buffer, for fixed of the processor speed as shown in Fig. 3.In particular, for the simulated emitter environment, when the deinterleaver's single 
comparison time with one radar chain is $35 \mu \mathrm{sec}$ and the pre-buffer size is 5 PDVs, then for arrival rates $\leq 2000 \mathrm{PDVs} / \mathrm{sec}$ the blocking probability is $\leq 50 \%$, (Fig. 2). Now, if the pre-buffer size is increased from zero to 80 PDVs, the deinterleaver can process up to $23400 \mathrm{PDVs} / \mathrm{sec}$ with blocking probability $\leq 50 \%$, (Fig. 3). Alternatively, if the single comparison time is decreased from $35 \mu \mathrm{sec}$ to $25 \mu \mathrm{sec}$, by increasing the processor speed ), the deinterleaver with pre-buffer size 5 PDVs can process up to 24000 PDVs/sec with blocking probability $\leq 50 \%$, (Fig. 2). Thus in our case, $40 \%$ increase of processor speed is roughly equivalent to 16 times increase of the pre-buffer size if the blocking probability is the measure of comparison. Although, fast processors are expensive they ensure high average services rates, minimal reporting latency of intercepted threats and are usually associated with less hardware (no need for large buffers). The single comparison time can also be decreased by optimizing the sorting algorithms and in some applications, by limiting the measurement of mono-pulse parameters in the ESM receiver-encoder only to those obtained from the pulse leading edge, (short PDVs).

\section{CONCLUSION}

In this paper, there has been analyzed the queueing behavior of an automatic ESM system in dense emitter environment. It has been concluded that an automatic ESM system can be generally represented as a cascade of two queueing models. The first queueing model describes the operation the ESM receiver-encoder subsystem and the second describes the operation of the deinterleaver processor. It is shown that the ESM receiver-encoder subsystem operates under either paralayzable or nonparalayzable service discipline. Analytic expressions for the ratio of successfully processed pulses have been derived under both service disciplines. The results show that for the same input arrival rate of radar pulses, the ratio of successfully processed pulses under non-paralayzable service discipline is higher than the same ratio under paralayzable service discipline. The departure process of PDVs from the receiverencoder subsystem has been analyzed for both service disciplines. It has been shown that the behavior of the deinterleaver is extremely improved when it is preceded by a pre-buffer. An analytic procedure has been defined for evaluation of the steady state blocking probability of the deinterleaver. The analysis of the queueing models describes the operation of automatic ESM system enables us to evaluate the probability of pulse loss due to: time overlapping, limited processing speed of the deinterleaver or insufficient storage capacity of the pre-puffer.

\section{REFERENCES}

[1] S. Curtis, Introduction to Electronic Warfare, Artech House, Inc., (1986).

[2]C. L. Davies And P. Hollands Comm., Radar \& Signal Process., 129, (3), pp. 146-171, (1982).

[3]J. B. Tsui, Microwave Receivers With Electronic Warfare Applications, J. Wiley \&Sons, (1986).

[4]S. Hillier And J. Lieberman, Introduction to Operation Research, Mcgrow-F:ill, $6^{\text {th }}$ Edition, (1995).

[5] D. Bunday, Basic Queuing Theory, Edward Arnold, (1986). 
Proceedings of the $\mathbf{2}^{\text {nd }}$ ICEENG Conference, 23-25 Nov. 1999

\begin{tabular}{|l|l|}
\hline RG-1 & 10 \\
\hline
\end{tabular}

[6]J. J. Bussgang

Interpulse Interval Distribution in The Environri?ent of $N$ Period RFI-5, pp. 7-10, (1963).

[7]Abou-Bakr, H., "Analysis of the Queueing Behavior of automatic ESM System in Dense Emitter Environments," Master of Science thesis in Electrical Engineering, M. T. C., Cairo, Egypt, (1998).

[8] C. Tijms, Stochastic Modeling and Analysis, J. Wiley \& Sons, (1986).

\section{Figure \& Table Captions}

-Fig.1:Comparison of ratios of successfully processed pulses under paralayzable and non-paralayzable counter service disciplines, PW is distributed according to Erlang distribution with $\bar{w}=1 / \mu=12 \mu \mathrm{sec}$ and for different $\theta$ : (a) $\theta=1$, (b) $\theta=2$, (c) $\theta=5$, (d) $\theta=8$, (e) $\theta=\infty$ (paralayzable).

-Fig. 2: Dependence of the deinterleaver blocking probability on the arrival rate of PDVs, constant pre-buffer size $=5$ PDVs, comparison time with one radar chain: (a) $45 \mu \mathrm{sec}$, (b) $35 \mu \mathrm{sec}$, (c) $25 \mu \mathrm{sec}$, (d) $15 \mu \mathrm{sec} . \mathrm{N}$ is the total number of active emitters within the instantaneous view.

-Fig. 3: Dependence of the deinterleaver blocking probability on the arrival rate of PDVs, constant comparison time with one radar chain $=35 \mu \mathrm{sec}$, : (a) pre-buffer size $=0$ PDV, (b) pre-buffer size =20 PDVs, (c) pre-buffer size $=40$ PDVs, (d) pre-buffer size $=80$ PDVs. $N$ is the total number of active emitters within the instantaneous view.

-Table 1: The relation between the arrival rate of radar pulses at the ESM receiver input and the sum of PRFs of radars illuminating the ESM receiver.

-Table 2: The square distance between the theoretical negative exponential distribution and the measured distribution of interarrival times between received radar pulses at different arrival rates.

-Table 3 : Comparison between the distribution of the number of received pulses during different time intervals and Poisson distribution with parameter $\lambda=30000$ pulse/sec: $N \equiv$ number of subintervals, $L \equiv$ width of subinterval, $P_{n_{t}}(L)=e^{-\lambda l}(\lambda L)^{n} / n !, P_{n_{e}}(L) \equiv$ estimated probability.

-Table 4 : Expressions for $\mu$ in four different cases.

-Table 5: The normalized squared distance between the theoretical delayed negative exponential distribution with parameters $\lambda$ and $\tau$ and the measured distribution of interdeparture times between output PDVs at different arrival rates [constant service time, $\tau=11 \mu \mathrm{sec}$.].

-Table 6:The normalized square distance between the theoretical negative exponential distribution with parameter $\lambda_{0}$ and measured distribution of the interdeparture times between output PDVs at different arrival rates [nonparalayzable counter service discipline, average $\mathrm{PW}=10 \mu \mathrm{sec}]$. 


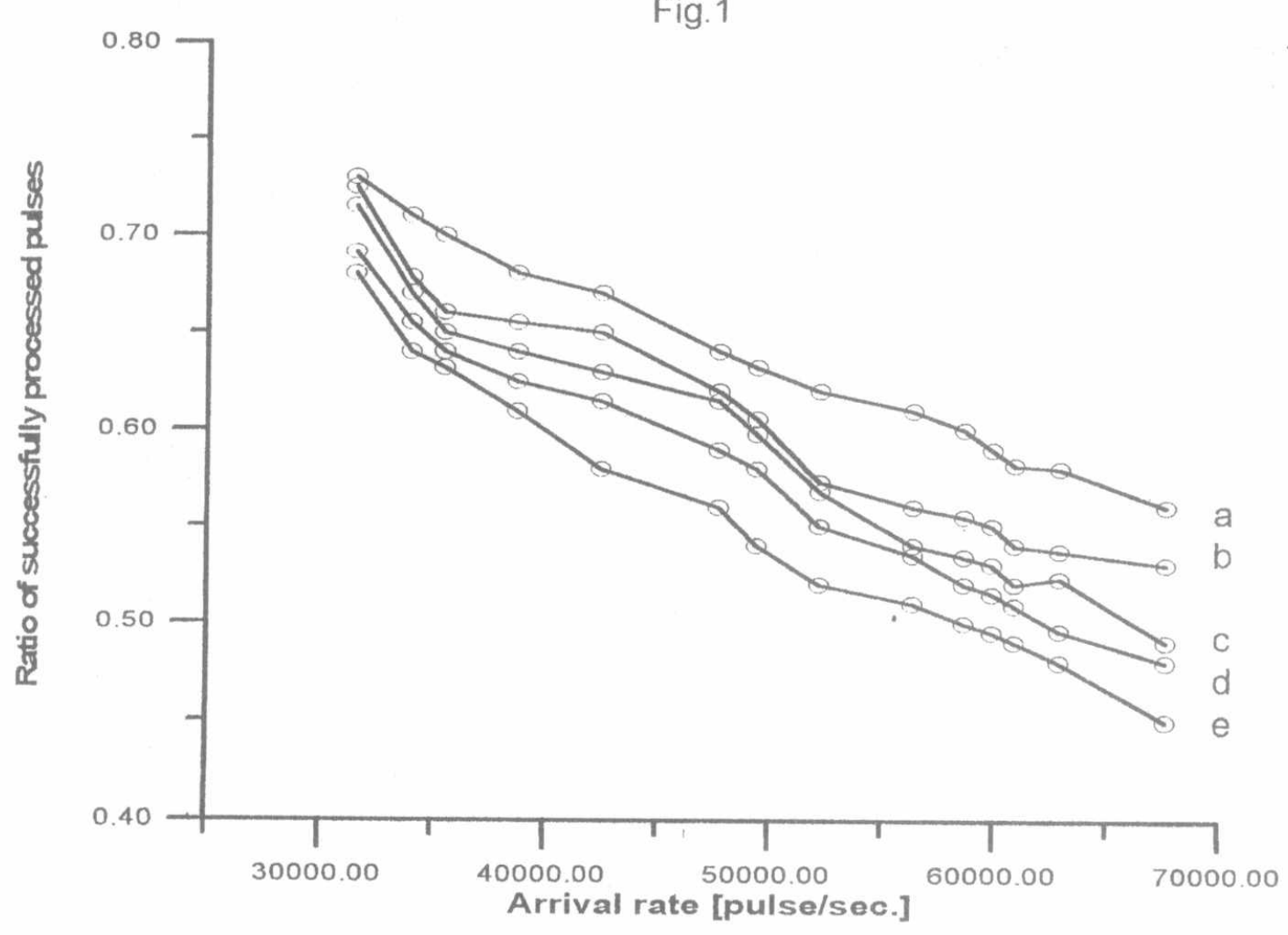

Fig. 2

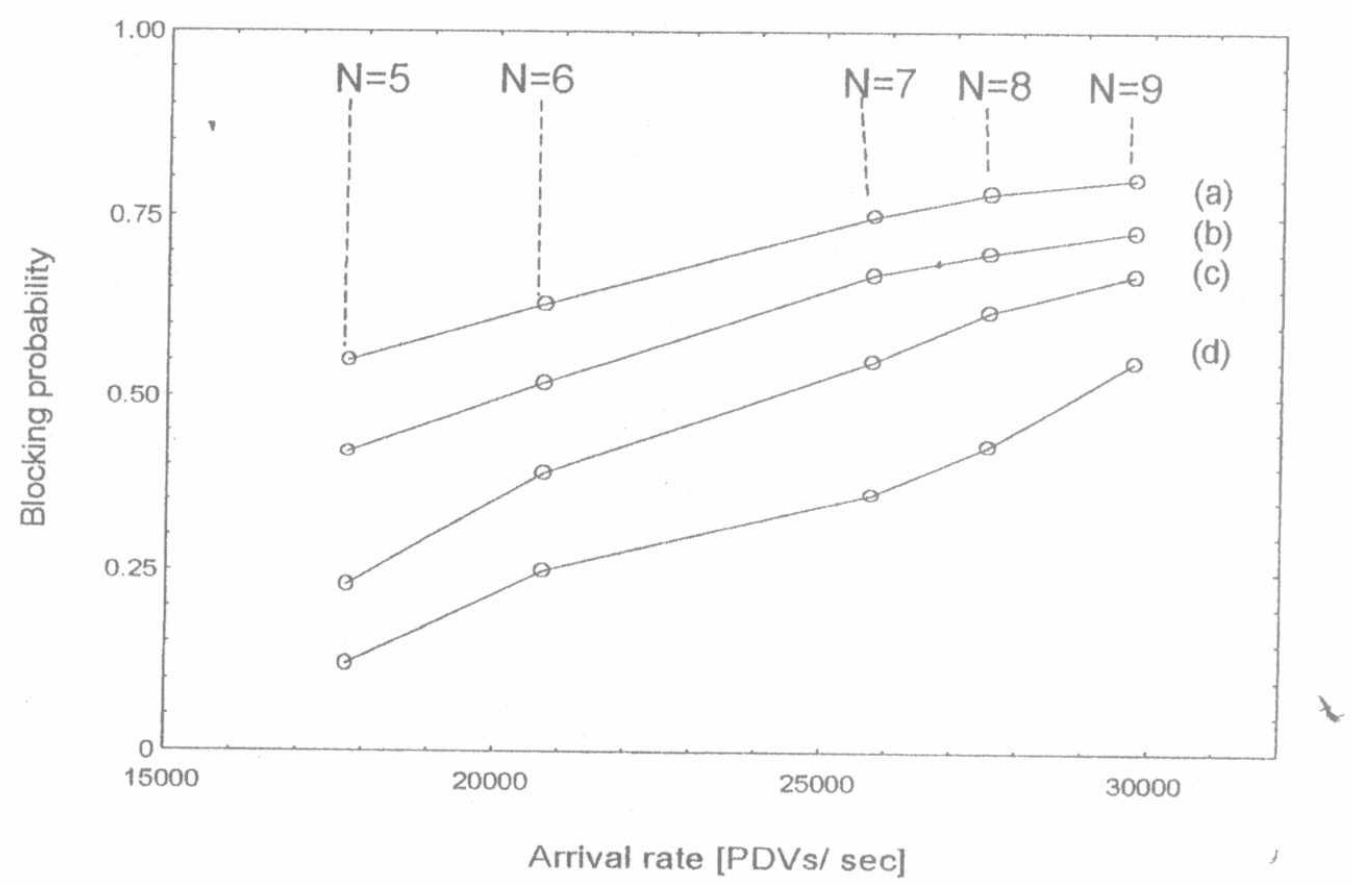


Proceedings of the $2^{\text {nd }}$ ICEENG Conference, 23-25 Nov. 1999

\begin{tabular}{|l|l|}
\hline RG-1 & 12 \\
\hline
\end{tabular}

Fig. 3

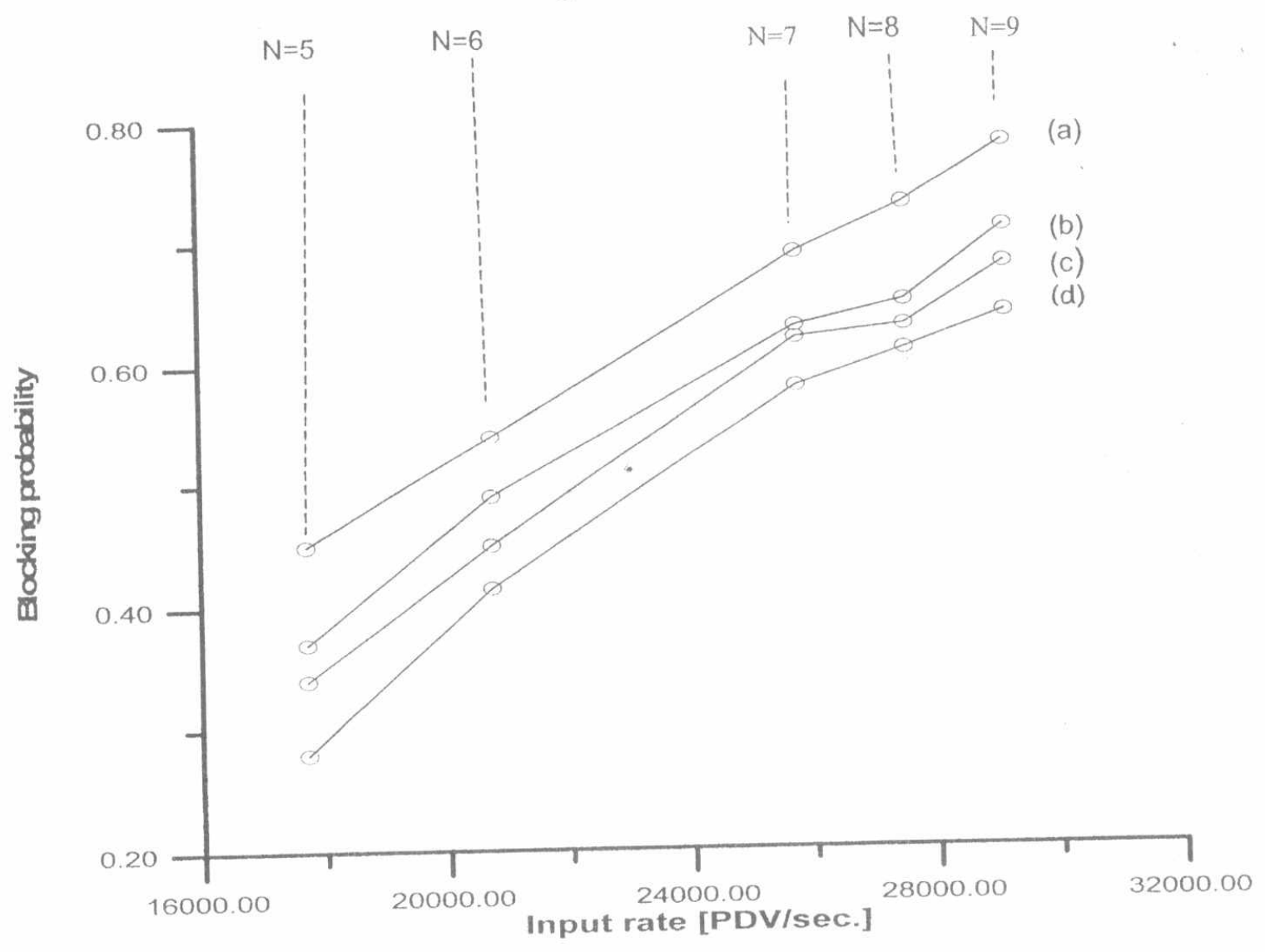

Table 1

\begin{tabular}{|l|l|l|}
\hline$\lambda=\sum_{i=1}^{N} P R F_{i}[$ Pulse/sec.] & $\begin{array}{l}\lambda_{\text {measured }} \\
{[\text { Pulse/sec. }}\end{array}$ & $\begin{array}{l}\text { Number of } \\
\text { active radars }\end{array}$ \\
\hline 31500 & 31485 & 7 \\
\hline 34000 & 33951 & 8 \\
\hline 35500 & 35441 & 9 \\
\hline 38750 & 38687 & 10 \\
\hline 42500 & 42439 & 11 \\
\hline 47750 & 47681 & 12 \\
\hline 49500 & 49388 & 13 \\
\hline 52250 & 52188 & 14 \\
\hline 56500 & 56362 & 15 \\
\hline 58750 & 58621 & 16 \\
\hline 60000 & 59857 & 17 \\
\hline 61000 & 60867 & 18 \\
\hline 63000 & 62860 & 19 \\
\hline
\end{tabular}


Proceedings of the $2^{\text {nd }}$ ICEENG Conference, 23-25 Nov. 1999

Table 2

\begin{tabular}{|l|l|l|l|l|l|l|l|l|l|}
\hline $\begin{array}{l}\lambda_{\text {measured }} \\
{[\mathrm{P} / \text { sec. }]}\end{array}$ & $\begin{array}{l}\mathrm{M}_{1 \mathrm{~m}} \\
\times 10^{5}\end{array}$ & $\begin{array}{l}\mathrm{M}_{1 \mathrm{t}} \\
\times 10^{5}\end{array}$ & $\begin{array}{l}\mathrm{M}_{2 \mathrm{~m}} \\
\times 10^{9}\end{array}$ & $\begin{array}{l}\mathrm{M}_{2 \mathrm{t}} \\
\times 10^{9}\end{array}$ & $\begin{array}{l}\mathrm{M}_{3 \mathrm{~m}} \\
\times 10^{13}\end{array}$ & $\begin{array}{l}\mathrm{M}_{3 \mathrm{t}} \\
\times 10^{13}\end{array}$ & $\begin{array}{l}\mathrm{M}_{4 \mathrm{~m}} \\
\times 10^{17}\end{array}$ & $\begin{array}{l}\mathrm{M}_{4 \mathrm{t}} \\
\times 10^{17}\end{array}$ & $\begin{array}{l}\mathrm{D}^{2} \\
\times 10^{6}\end{array}$ \\
\hline 31485 & 3.17 & 3.17 & 2.09 & 2.05 & 2.10 & 1.90 & 2.80 & 2.40 & 2.20 \\
\hline 33951 & 2.90 & 2.90 & 1.90 & 1.70 & 1.90 & 1.70 & 2.60 & 1.70 & 2.06 \\
\hline 35441 & 2.80 & 2.80 & 1.86 & 1.50 & 1.89 & 1.34 & 2.50 & 1.50 & 0.39 \\
\hline 38687 & 2.50 & 2.50 & 1.50 & 1.33 & 1.50 & 1.03 & 1.30 & 1.00 & 0.26 \\
\hline 42439 & 2.30 & 2.30 & 1.30 & 1.01 & 1.16 & 0.78 & 1.40 & 0.73 & 2.05 \\
\hline 47681 & 2.09 & 2.09 & 1.07 & 0.87 & 0.83 & 0.55 & 0.99 & 0.46 & 2.00 \\
\hline 49388 & 2.02 & 2.02 & 0.98 & 0.82 & 0.81 & 0.45 & 0.95 & 0.39 & 3.30 \\
\hline 52138 & 1.95 & 1.95 & 0.43 & 0.73 & 0.79 & 0.42 & 0.90 & 0.32 & 1.65 \\
\hline 56362 & 1.77 & 1.76 & 0.82 & 0.63 & 0.66 & 0.33 & 0.81 & 0.23 & 5.90 \\
\hline 58621 & 1.70 & 1.75 & 0.78 & 0.57 & 0.63 & 0.29 & 0.78 & 0.20 & 4.70 \\
\hline 59857 & 1.67 & 1.66 & 0.76 & 0.55 & 0.63 & 0.27 & 0.76 & 0.18 & 2.89 \\
\hline 60867 & 1.64 & 1.63 & 0.75 & 0.53 & 0.61 & 0.26 & 0.75 & 0.17 & 4.70 \\
\hline 62860 & 1.59 & 1.58 & 0.72 & 0.51 & 0.60 & 0.23 & 0.72 & 0.15 & 4.30 \\
\hline 67590 & 1.47 & 1.47 & 0.62 & 0.43 & 0.51 & 0.19 & 0.65 & 0.11 & 5.54 \\
\hline
\end{tabular}

Table 3

\begin{tabular}{|l|l|l|}
\hline $\mathrm{N}=2100, \mathrm{~L}=100 \mu \mathrm{sec}$. \\
\hline $\mathrm{n}$ & $P_{n_{t}}(L)$ & $P_{n_{e}}(L)$ \\
& & \\
\hline 0 & 0.049 & 0.046 \\
\hline 1 & 0.149 & 0.160 \\
\hline 2 & 0.224 & 0.229 \\
\hline 3 & 0.224 & 0.242 \\
\hline 4 & 0.168 & 0.170 \\
\hline 5 & 0.101 & 0.120 \\
\hline 6 & 0.050 & 0.045 \\
\hline & & \\
\hline
\end{tabular}

Most likely values occur at $n=\lambda L$

Table 4

\begin{tabular}{|l|l|l|l|l|}
\hline & $\begin{array}{l}\text { Service } \\
\text { discipline }\end{array}$ & $\begin{array}{l}\text { Service time } \\
\text { Distribution }\end{array}$ & $\begin{array}{l}\text { Queuein } \\
\text { g Model }\end{array}$ & $\mu$ \\
\hline Case 1 & Paralayzable & Fixed value $\tau$ & $\mathrm{M} / \mathrm{D} / 1 / 0$ & $\lambda e^{-\lambda \tau}$ \\
\hline Case 2 & $\begin{array}{l}\text { Non- } \\
\text { paralayzable }\end{array}$ & $\begin{array}{l}\text { Exponential with } \\
\text { mean value }=\bar{w}(1)\end{array}$ & $\mathrm{M} / \mathrm{M} / 1 / 0$ & $\frac{\lambda}{1+\lambda \bar{w}}$ \\
\hline Case 3 & $\begin{array}{l}\text { Non- } \\
\text { paralayzable }\end{array}$ & $\begin{array}{l}\text { Erlang with parameter } \\
\text { and mean } \bar{w}\end{array}$ & $\mathrm{M} / \mathrm{E}_{0} / 1 / 0$ & $\lambda\left(1+\frac{\lambda \bar{w}}{\theta}\right)^{-\theta}$ \\
\hline Case 4 & $\begin{array}{l}\text { Non- } \\
\text { paralayzable }\end{array}$ & $\begin{array}{l}\text { Uniform in } \\
{\left[\mathrm{w}_{\min }, \mathrm{w}_{\max }\right]^{(2)}}\end{array}$ & $\mathrm{M} / \mathrm{G} / 1 / 0$ & $\frac{e^{-\lambda w_{\min }}-e^{-\lambda w_{\max }}}{\left(w_{\max }-w_{\min }\right)}$ \\
\hline
\end{tabular}

(1) $\bar{w}$ is mean width of arriving pulses.

${ }^{\text {(2) }} \mathrm{W}_{\min }$ and $\mathrm{W}_{\max }$ are maximum and minimum widths of arriving pulses respectively. 
Proceedings of the $2^{\text {nd }}$ ICEENG Conference, 23-25 Nov. 1999

\begin{tabular}{|l|l|}
\hline RG-1 & 14 \\
\hline
\end{tabular}

Table 5

\begin{tabular}{|l|l|l|l|l|l|l|l|l|l|}
\hline $\begin{array}{l}\lambda_{\text {measured }} \\
{[\mathrm{P} / \text { sec. }]}\end{array}$ & $\begin{array}{l}\mathrm{M}_{1 \mathrm{~m}} \\
\times 10^{5}\end{array}$ & $\begin{array}{l}\mathrm{M}_{1 \mathrm{t}} \\
\times 10^{5}\end{array}$ & $\begin{array}{l}\mathrm{M}_{2 \mathrm{~m}} \\
\times 10^{9}\end{array}$ & $\begin{array}{l}\mathrm{M}_{2 \mathrm{t}} \\
\times 10^{9}\end{array}$ & $\begin{array}{l}\mathrm{M}_{3 \mathrm{~m}} \\
\times 10^{13}\end{array}$ & $\begin{array}{l}\mathrm{M}_{3 \mathrm{t}} \\
\times 10^{13}\end{array}$ & $\begin{array}{l}\mathrm{M}_{4 \mathrm{~m}} \\
\times 10^{17}\end{array}$ & $\begin{array}{l}\mathrm{M}_{4 \mathrm{t}} \\
\times 10^{17}\end{array}$ & $\begin{array}{l}D^{2} \\
\times 10^{4}\end{array}$ \\
\hline 31485 & 4.30 & 4.27 & 2.50 & 2.25 & 2.73 & 2.70 & 3.49 & 3.44 & 0.49 \\
\hline 33951 & 4.03 & 4.04 & 2.36 & 2.15 & 2.02 & 2.21 & 2.68 & 2.60 & 0.66 \\
\hline 35441 & 3.88 & 3.90 & 2.33 & 2.13 & 2.10 & 1.90 & 2.58 & 2.40 & 1.09 \\
\hline 38687 & 3.70 & 3.68 & 2.24 & 2.02 & 1.74 & 1.50 & 1.75 & 1.60 & $0.2 \mathrm{~s}$ \\
\hline 42439 & 3.46 & 3.45 & 2.06 & 1.74 & 1.52 & 1.24 & 1.27 & 1.17 & 0.84 \\
\hline 47681 & 3.25 & 3.19 & 1.78 & 1.46 & 1.27 & 0.93 & 1.19 & 0.78 & 0.48 \\
\hline 49388 & 3.20 & 3.12 & 1.72 & 1.38 & 1.29 & 0.85 & 1.08 & 0.69 & 6.57 \\
\hline 52138 & 3.05 & 3.01 & 1.71 & 1.27 & 0.92 & 0.79 & 1.04 & 0.57 & 1.12 \\
\hline 56362 & 2.90 & 2.85 & 1.56 & 1.19 & 0.85 & 0.62 & 1.01 & 0.44 & 3.07 \\
\hline 58621 & 2.84 & 2.80 & 1.02 & 1.07 & 0.80 & 0.61 & 0.97 & 0.38 & 2.04 \\
\hline 59857 & 2.90 & 2.77 & 1.38 & 1.04 & 1.13 & 0.53 & 0.88 & 0.36 & 1.28 \\
\hline 62860 & 2.85 & 2.65 & 1.30 & 0.97 & 1.05 & 0.47 & 0.75 & 0.31 & 3.50 \\
\hline
\end{tabular}

Table 6

\begin{tabular}{|l|l|l|l|l|l|l|l|l|l|}
\hline $\begin{array}{l}\lambda_{\text {measured }} \\
{[\mathrm{P} / \mathrm{sec}]}\end{array}$ & $\begin{array}{l}\mathrm{M}_{1 \mathrm{~m}} \\
\times 10^{5}\end{array}$ & $\begin{array}{l}\mathrm{M}_{1 \mathrm{t}} \\
\times 10^{5}\end{array}$ & $\begin{array}{l}\mathrm{M}_{2 \mathrm{~m}} \\
\times 10^{9}\end{array}$ & $\begin{array}{l}\mathrm{M}_{2 \mathrm{t}} \\
\times 10^{9}\end{array}$ & $\begin{array}{l}\mathrm{M}_{3 \mathrm{~m}} \\
\times 10^{13}\end{array}$ & $\begin{array}{l}\mathrm{M}_{3 \mathrm{t}} \\
\times 10^{13}\end{array}$ & $\begin{array}{l}\mathrm{M}_{4 \mathrm{~m}} \\
\times 10^{17}\end{array}$ & $\begin{array}{l}\mathrm{M}_{4 \mathrm{t}} \\
\times 10^{17}\end{array}$ & $\begin{array}{l}\mathrm{D}^{2} \\
\times 10^{4}\end{array}$ \\
\hline 31485 & 4.07 & 4.17 & 2.50 & 3.40 & 2.55 & 4.33 & 3.31 & 7.20 & 5.90 \\
\hline 33951 & 4.01 & 3.90 & 2.55 & 3.11 & 2.50 & 3.68 & 3.20 & 5.80 & 3.16 \\
\hline 35441 & 4.02 & 3.80 & 2.50 & 2.90 & 2.50 & 3.30 & 3.20 & 5.10 & 2.40 \\
\hline 38687 & 3.60 & 3.50 & 2.10 & 2.50 & 1.90 & 2.70 & 2.40 & 3.90 & 0.74 \\
\hline 42439 & 3.30 & 3.33 & 1.79 & 2.25 & 1.50 & 2.26 & 1.50 & 3.17 & 0.05 \\
\hline 47681 & 3.05 & 3.09 & 1.45 & 1.91 & 1.14 & 1.71 & 1.30 & 2.20 & 5.80 \\
\hline 49388 & 2.99 & 3.02 & 1.39 & 1.83 & 1.09 & 1.66 & 1.20 & 2.17 & 0.93 \\
\hline 52138 & 2.88 & 2.95 & 1.30 & 1.70 & 1.05 & 1.49 & 1.20 & 1.70 & 11.00 \\
\hline 56362 & 2.69 & 2.70 & 1.18 & 1.50 & 0.93 & 1.28 & 1.10 & 1.40 & 8.00 \\
\hline 58621 & 2.65 & 2.70 & 1.14 & 1.46 & 0.90 & 1.18 & 1.10 & 1.20 & 9.00 \\
\hline 59857 & 2.63 & 2.67 & 1.20 & 1.42 & 0.90 & 1.14 & 0.95 & 1.10 & 0.31 \\
\hline 60867 & 2.62 & 2.64 & 1.16 & 1.39 & 0.90 & 1.10 & 0.92 & 1.10 & 0.02 \\
\hline 62860 & 2.60 & 2.59 & 1.14 & 1.34 & 0.90 & 1.04 & 1.00 & 1.08 & 0.82 \\
\hline 67590 & 2.45 & 2.47 & 1.02 & 1.22 & 0.82 & 0.91 & 0.95 & 0.90 & 0.65 \\
\hline
\end{tabular}

